\title{
Response to: Comment on "Exotropia Is the Main Pattern of Childhood Strabismus Surgery in the South of China: A Six-Year Clinical Review"
}

\author{
Xinping Yu, ${ }^{1}$ Zhouduo Ji, ${ }^{2}$ Huanyun Yu, ${ }^{1}$ Meiping Xu, ${ }^{1}$ and Jinling $X u^{1}$ \\ ${ }^{1}$ The Eye Hospital of Wenzhou Medical University, Wenzhou 325027, China \\ ${ }^{2}$ Ophthalmology Department, Zhengzhou Second Hospital, Zhengzhou 450006, China \\ Correspondence should be addressed to Xinping Yu; yu-xinping@163.com
}

Received 19 October 2016; Accepted 8 November 2016; Published 16 March 2017

Academic Editor: Lisa Toto

Copyright (C) 2017 Xinping Yu et al. This is an open access article distributed under the Creative Commons Attribution License, which permits unrestricted use, distribution, and reproduction in any medium, provided the original work is properly cited.

Thanks are due to Dr. Ayyildiz et al. [1] for the constructive questions and suggestions to our publication [2]. We included our answers below.

The distributions of strabismus subtypes for all age groups between 2006 and 2011 were listed in Table 1.

The surgical criteria for intermittent exotropia were the same for all patients included in the study, which included (1) the degree of deviation (distance and/or near) $\geqq 20 \mathrm{PD}$ and (2) the frequency of deviation occurring more than $1 / 2$ of waking hours, and/or (3) those combined with sensory deficit $[3,4]$. Here the sensory deficit included the stereoacuity at near and distance, binocular vision measured through stereoscope, and the sensory fusion measured by Worth 4 Dot test.

And the surgical criteria for esotropia in children are mainly based on the effect of deviation with wearing corrected glasses, in patients still with deviation more than 10 $\mathrm{PD}$ at near or distance with the corrected glasses [5].

We did evaluate the stereoacuity at distance using Optec 3500 in the study. 722 (32.5\%) subjects had 40-480 seconds of arc at distance, $996(44.8 \%)$ had $>480$ seconds of arc, and $505(22.7 \%)$ were uncooperative to the test.

We hope we have addressed all questions and made our result clearer. 
TABLE 1: Distributions of strabismus subtypes for all age groups between 2006 and 2011.

\begin{tabular}{|c|c|c|c|c|c|c|c|c|c|c|c|c|}
\hline \multirow{3}{*}{ Age group } & \multicolumn{12}{|c|}{ Year } \\
\hline & \multicolumn{2}{|c|}{2006} & \multicolumn{2}{|c|}{2007} & \multicolumn{2}{|c|}{2008} & \multicolumn{2}{|c|}{2009} & \multicolumn{2}{|c|}{2010} & \multicolumn{2}{|c|}{2011} \\
\hline & $0-6$ & $7-17$ & $0-6$ & $7-17$ & $0-6$ & $7-17$ & $0-6$ & $7-17$ & $0-6$ & $7-17$ & $0-6$ & $7-17$ \\
\hline \multirow{2}{*}{ Intermittent exotropia } & 6 & 45 & 5 & 36 & 15 & 67 & 29 & 78 & 31 & 88 & 40 & 115 \\
\hline & $(10 \%)$ & $(24 \%)$ & $(6 \%)$ & $(17 \%)$ & $(16 \%)$ & $(25 \%)$ & $(28 \%)$ & $(29 \%)$ & $(24 \%)$ & $(29 \%)$ & $(25 \%)$ & $(33 \%)$ \\
\hline \multirow{2}{*}{ Constant exotropia } & 15 & 27 & 8 & 57 & 10 & 51 & 17 & 71 & 13 & 49 & 14 & 61 \\
\hline & $(23 \%)$ & $(15 \%)$ & $(9 \%)$ & $(28 \%)$ & $(11 \%)$ & $(19 \%)$ & $(16 \%)$ & $(26 \%)$ & $(10 \%)$ & $(16 \%)$ & $(9 \%)$ & $(17 \%)$ \\
\hline \multirow{2}{*}{ Infant esotropia } & 3 & 9 & 14 & 7 & 8 & 6 & 7 & 5 & 10 & 14 & 15 & 13 \\
\hline & $(5 \%)$ & $(5 \%)$ & $(16 \%)$ & $(3 \%)$ & $(8 \%)$ & $(2 \%)$ & $(6.5 \%)$ & $(2 \%)$ & $(8 \%)$ & $(5 \%)$ & $(9 \%)$ & $(4 \%)$ \\
\hline \multirow{2}{*}{ Partial accommodative esotropia } & 4 & 12 & 10 & 16 & 9 & 18 & 10 & 11 & 9 & 15 & 16 & 20 \\
\hline & $(6 \%)$ & $(6 \%)$ & $(12 \%)$ & $(8 \%)$ & $(9 \%)$ & $(7.5 \%)$ & $(10 \%)$ & $(4 \%)$ & $(7 \%)$ & $(5 \%)$ & $(10 \%)$ & $(6 \%)$ \\
\hline \multirow{2}{*}{ Nonaccommodative esotropia } & 9 & 30 & 14 & 35 & 20 & 48 & 18 & 35 & 14 & 49 & 21 & 40 \\
\hline & $(14 \%)$ & $(16 \%)$ & $(16 \%)$ & $(17 \%)$ & $(21 \%)$ & $(18 \%)$ & $(17 \%)$ & $(13 \%)$ & $(11 \%)$ & $(16 \%)$ & $(13 \%)$ & $(12 \%)$ \\
\hline \multirow{2}{*}{ Congenital superior oblique palsy } & 8 & 8 & 10 & 6 & 14 & 16 & 8 & 12 & 14 & 13 & 13 & 20 \\
\hline & $(12 \%)$ & $(4 \%)$ & $(12 \%)$ & $(3 \%)$ & $(15 \%)$ & $(6.5 \%)$ & $(7.5 \%)$ & $(4 \%)$ & $(11 \%)$ & $(4 \%)$ & $(8 \%)$ & $(6 \%)$ \\
\hline \multirow{2}{*}{ Others } & 19 & 55 & 25 & 50 & 19 & 58 & 16 & 60 & 37 & 76 & 42 & 78 \\
\hline & $(30 \%)$ & $(30 \%)$ & $(29 \%)$ & $(24 \%)$ & $(20 \%)$ & $(22 \%)$ & $(15 \%)$ & $(22 \%)$ & $(29 \%)$ & $(25 \%)$ & $(26 \%)$ & $(22 \%)$ \\
\hline \multirow{2}{*}{ Total } & 64 & 186 & 86 & 207 & 95 & 264 & 105 & 272 & 128 & 304 & 161 & 347 \\
\hline & $(100 \%)$ & $(100 \%)$ & $(100 \%)$ & $(100 \%)$ & $(100 \%)$ & $(100 \%)$ & $(100 \%)$ & $(100 \%)$ & $(100 \%)$ & $(100 \%)$ & $(100 \%)$ & $(100 \%)$ \\
\hline
\end{tabular}

\section{Competing Interests}

The authors declare no financial nor ethical/legal conflict of interests related to the paper.

\section{References}

[1] O. Ayyildiz, O. M. Ceylan, and F. Mehmet Mutlu, "Comment on 'Exotropia is the main pattern of childhood strabismus surgery in the south of china: a six-year clinical review"' Journal of Ophthalmology, vol. 2016, Article ID 8251629, 1 page, 2016.

[2] X. Yu, Z. Ji, H. Yu, M. Xu, and J. Xu, "Exotropia is the main pattern of childhood strabismus surgery in the south of China: a six-year clinical review," Journal of Ophthalmology, vol. 2016, Article ID 1489537, 5 pages, 2016.

[3] K. W. Wright, "Extropia," in Color Atlas of Strabismus Surgery Strategies and Techniques, p. 45, Springer Science+Business Media, LLC, 2007.

[4] S. R. Hatt and L. Gnanaraj, "Interventions for intermittent exotropia (Review)," Cochrane Database of Systematic Reviews, Article ID D003737, 2006.

[5] W. K. Wright, "Acquired esotropia, infant esotropia," in Color Atlas of Strabismus Surgery Strategies and Techniques, pp. 18-40, Springer, Berlin, Germany, 2007. 


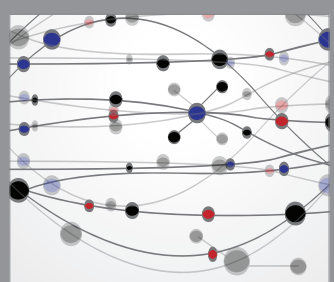

The Scientific World Journal
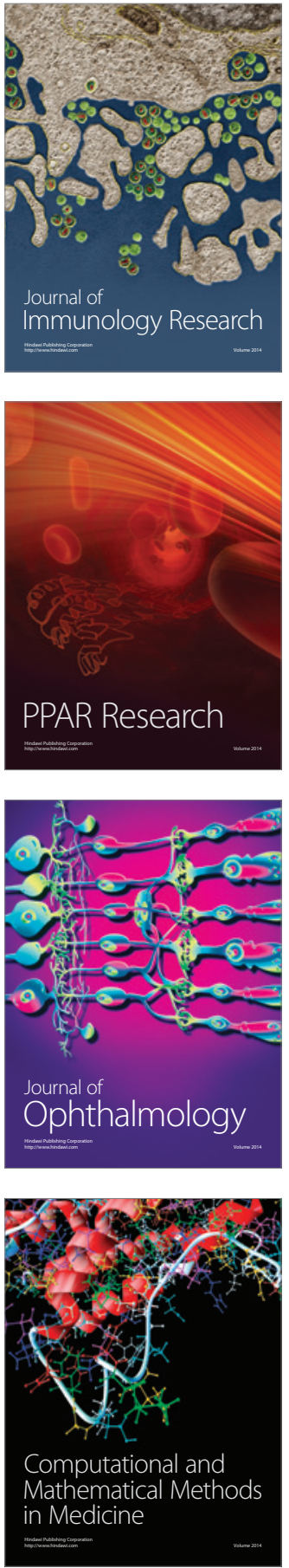

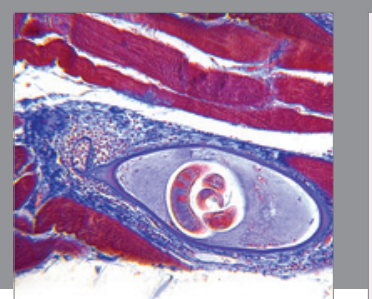

Gastroenterology Research and Practice
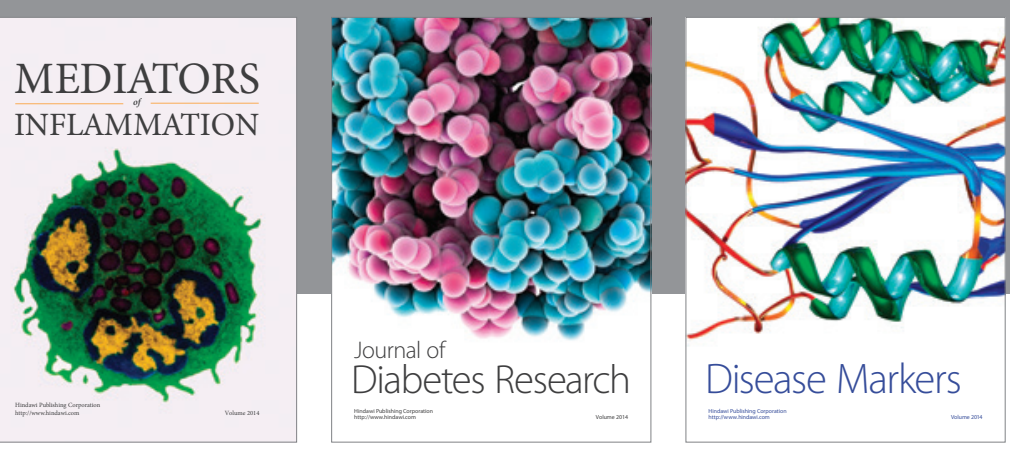

Disease Markers

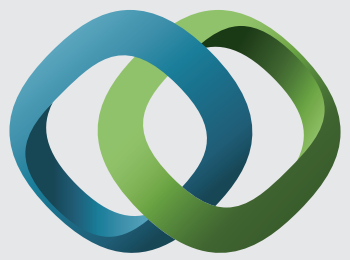

\section{Hindawi}

Submit your manuscripts at

https://www.hindawi.com
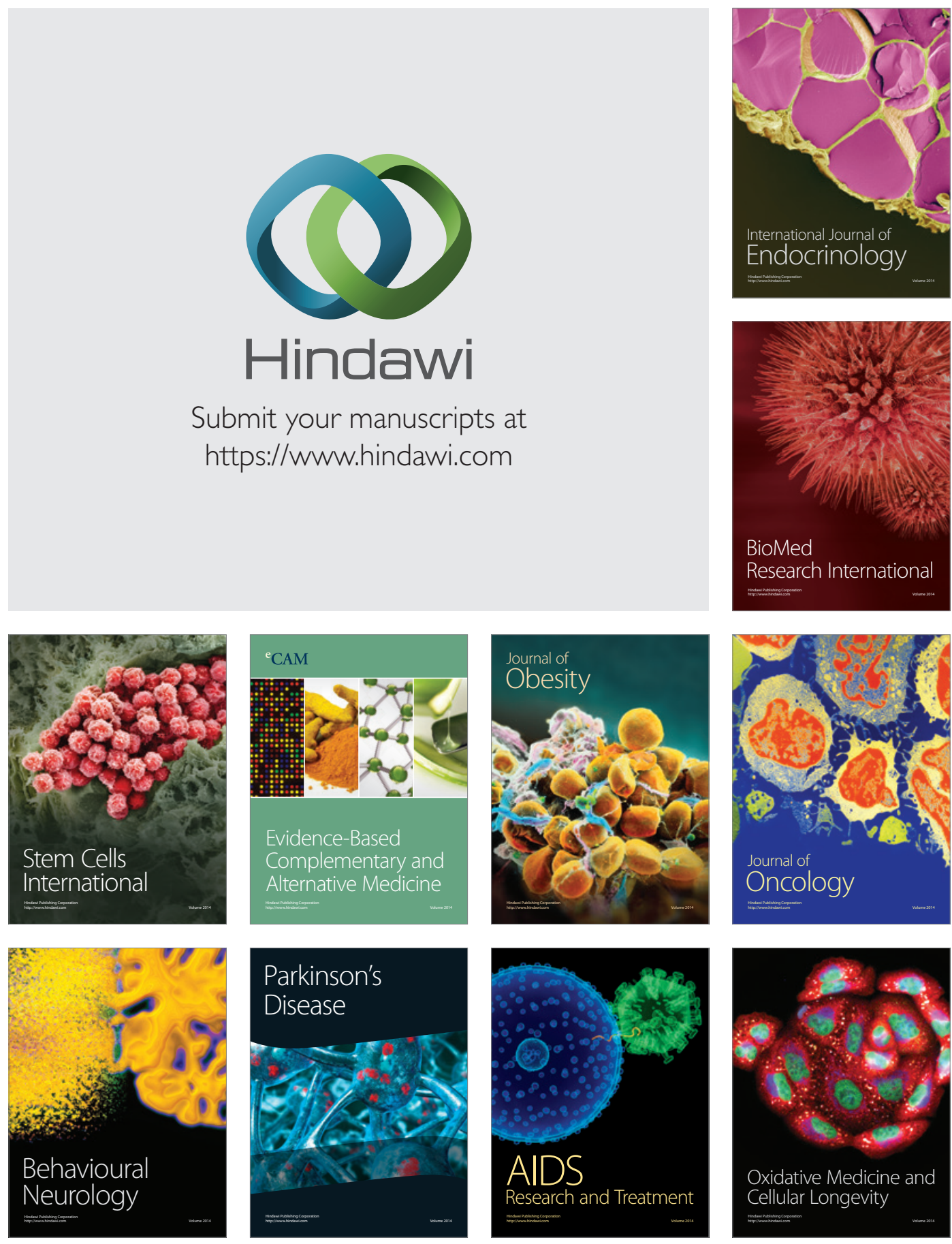